\title{
Estudio Teórico sobre la Hidrólisis Ácida de Amidas Alifáticas y Aromáticas
}

\author{
Silvana C. Caglieri y Mariángeles Pagnan \\ Universidad Tecnológica Nacional, Facultad Regional Córdoba, CIQA-Centro de Investigación y \\ Transferencia en Ingeniería Química Ambiental, Departamento de Ingeniería Química, Av. Cruz Roja \\ Argentina esquina Maestro López. Ciudad Universitaria (X5016ZAA). Córdoba-Argentina. \\ (e-mail: scaglieri@quimica.frc.utn.edu.ar ; mpagnan@quimica.frc.utn.edu.ar)
}

Recibido Dic. 21, 2012; Aceptado Feb. 11, 2013; Versión final recibida Feb. 14, 2013

\begin{abstract}
Resumen
Se realizó un estudio teórico comparativo de la reactividad de las amidas alifáticas Acetamida y $\mathrm{N}$ metilacetamida y de las amidas aromáticas Benzamida y N-metilbenzamida, frente a la reacción de hidrólisis catalizada por ácido. Esto se hace a través del análisis de los intermediarios de reacción. Se modelaron y determinaron las geometrías de todas las especies que intervienen en la hidrólisis y se calcularon los parámetros geométricos y las energías de los reactivos y productos, empleando dos métodos de estructura electrónica, teoría del funcional de la densidad (DFT) y el modelo Austin (AM1). Los valores obtenidos se compararon con datos experimentales y de literatura observándose una gran concordancia. Siguiendo el mismo procedimiento se determinaron los parámetros geométricos y energías de los intermediarios de reacción, observándose una mayor estabilidad para el intermediario alifático, que reportó una energía inferior comparada con la del aromático.
\end{abstract}

Palabras clave: hidrólisis ácida, amida alifática, amida aromática, teoría del funcional de la densidad, modelo Austin

\section{Theoretical Study on the Acid Hydrolysis of Aliphatic and Aromatic Amides}

\begin{abstract}
A comparative theoretical study of reactivity of the aliphatic amides Acetamide and $\mathrm{N}$-methylacetamide and of the aromatic amides Benzamide and $\mathrm{N}$-methylbenzamide for the hydrolysis reaction catalyzed by acid. This is done through the analysis of intermediates of reactions. The geometry of all species involved in the hydrolysis was determined and the energies of all reagents and products were calculated. Two methods of electronic structure, density functional theory (DFT) and the Austin Model 1 (AM1) were used. The calculated values were compared with experimental and literature data. Following the same procedure, the geometric parameters and energies of reaction intermediates were determined, observing greater stability for the aliphatic intermediate, with lower energy compared with the aromatic substances.
\end{abstract}

Keywords: acid hydrolysis, aliphatic amide, aromatic amide, density functional theory, Austin Model 


\section{INTRODUCCIÓN}

El estudio teórico de la hidrólisis ácida de las amidas alifáticas y aromáticas, cuyos productos de reacción consisten en aminas y ácidos orgánicos, es de gran interés, no sólo por la utilidad de estos últimos dentro de la industria química (Morero y Campanella, 2013), sino que desde el punto de vista ambiental, dicha reacción constituye una vía para la degradación de poliamidas.

Han sido reportados estudios sobre síntesis y caracterización de poliamidas biodegradables (Wang et al., 2009) e investigaciones sobre el mecanismo de reacción de la hidrólisis de poliamidas sintéticas (Jacques et al., 2002), catalizada por álcalis, ácidos y en agua pura, como método para la degradación de enlaces peptídicos.

Las amidas se hidrolizan lentamente ya que poseen grupos salientes muy básicos y además el agua es un nucleófilo débil. El aumento de la velocidad de la hidrólisis se puede conseguir con el agregado de un ácido como catalizador. Para que un catalizador aumente la velocidad de una reacción debe aumentar la velocidad del paso lento o paso determinante de la misma. La hidrólisis es una reacción de sustitución nucleofílica sobre carbono insaturado, el ácido que actúa como catalizador, primero protona el oxígeno del carbonilo y posteriormente se produce el ataque nucleofílico del agua para formar el intermediario tetraédrico, etapa determinante de la velocidad de reacción. El ácido aumenta la velocidad de formación del intermediario tetraédrico al protonar el oxígeno del carbonilo, porque un grupo carbonilo protonado es más susceptible de ser atacado por un nucleófilo que un grupo carbonilo no protonado.

Se han llevado a cabo estudios teóricos sobre la hidrólisis de amidas catalizada con ácidos (Hori et al., 2007) y con bases (Xiong y Zhan, 2006), mediante la aplicación de diversos métodos computacionales para estudiar la cinética de dicha reacción. Tanto estos estudios, como trabajos experimentales (Cheshmedzhieva et al., 2009) concuerdan en que dicha reacción transcurre a través de la formación de un intermediario tetraédrico.

En este artículo se presenta un estudio teórico comparativo de la hidrólisis ácida de Acetamida, Benzamida, $\mathrm{N}$-metilacetamida y $\mathrm{N}$-metilbenzamida, cuyos productos de reacción son acetato y benzoato de amonio para las dos primeras amidas y metil amina y los ácidos acético y benzoico respectivamente para las dos últimas amidas enunciadas, empleando la Química Teórica Computacional como herramienta para estudiar las estructuras y propiedades de compuestos orgánicos y predecir comportamientos de reacciones químicas.

\section{DESARROLLO DEL TEMA}

Para poder comparar la reactividad de las amidas frente a la hidrólisis, se diseñaron y optimizaron las estructuras de todas las especies que intervienen en las reacciones, determinándose los parámetros geométricos óptimos correspondientes, calculando además las energías mínimas de todos los compuestos, reactivos y productos, que participan en las reacciones como así también la energía de los respectivos intermediarios.

Para realizar estos cálculos se emplearon métodos de estructura electrónica. Estos métodos dentro de la química computacional, utilizan las leyes de la mecánica cuántica como base para los cálculos, mediante la resolución de la ecuación de Schrödinger, que independiente del tiempo toma la forma de la siguiente ecuación:

$H \Psi_{(r)}=E \Psi_{(r)}$

Siendo $\mathrm{H}$ una función diferencial que incluye la energía cinética y potencial de núcleos y electrones, denominada operador hamiltoniano, $\Psi$ la función de onda molecular y $E$ energía. La solución que obtiene el menor valor de energía es la que corresponde al estado basal.

Dentro de los métodos de estructura electrónica, se empleó el método DFT basado en la teoría de funcionales de densidad (Parr y Yang, 1989), el cual incluye efectos de correlación electrónica y dentro de este se empleó el B3LYP (Becke, 1997). Este método denominado ab-initio, considera toda la estructura atómica sin introducir parámetros empíricos en la resolución de la ecuación de Schrödinger. Se optó por la función de base $3-21 \mathrm{G}^{*}$ que indica que se utilizan tres funciones gaussianas para representar los orbitales atómicos internos, mientras que los externos, capa de valencia, se representan mediante dos funciones para la parte contraída y una para la parte difusa, más una función de polarización para átomos pesados, indicada mediante el ${ }^{*}$, que representa el efecto de los orbitales tipo d. 
También para calcular las energías mínimas de todas las moléculas que intervienen en ambas reacciones se empleó el método semiempírico AM1, Austin Model 1 (Dewart et al., 1985), que utiliza parámetros derivados de resultados experimentales para simplificar los cálculos cuánticos y reducir su coste computacional. Este método omite los cálculos relativos a los electrones de capa interna, que los trata como si formasen parte del núcleo y se centra únicamente en los electrones externos, considerando a éstos electrones los responsables de la reactividad. Todos los cálculos se realizaron empleando el programa Gaussian03 (Frisch et al., 2004).

\section{RESULTADOS Y DISCUSIÓN}

En la Fig.1 se describen las etapas a través de las cuales se lleva a cabo la hidrólisis ácida de Acetamida $\left(R=\mathrm{CH}_{3}\right.$ y $\left.\mathrm{R}_{1}=\mathrm{H}\right)$, N-metilacetamida $\left(\mathrm{R}=\mathrm{CH}_{3}\right.$ y $\left.\mathrm{R}_{1}=\mathrm{CH}_{3}\right)$, Benzamida $\left(\mathrm{R}=\mathrm{C}_{6} \mathrm{H}_{5}\right.$ y $\left.\mathrm{R}=\mathrm{H}\right)$ y $\mathrm{N}$-metilbenzamida $\left(\mathrm{R}=\mathrm{C}_{6} \mathrm{H}_{5}\right.$ y $\left.\mathrm{R}_{1}=\mathrm{CH}_{3}\right)$, siendo estas reacciones reversibles.<smiles>[R][I-]N</smiles>
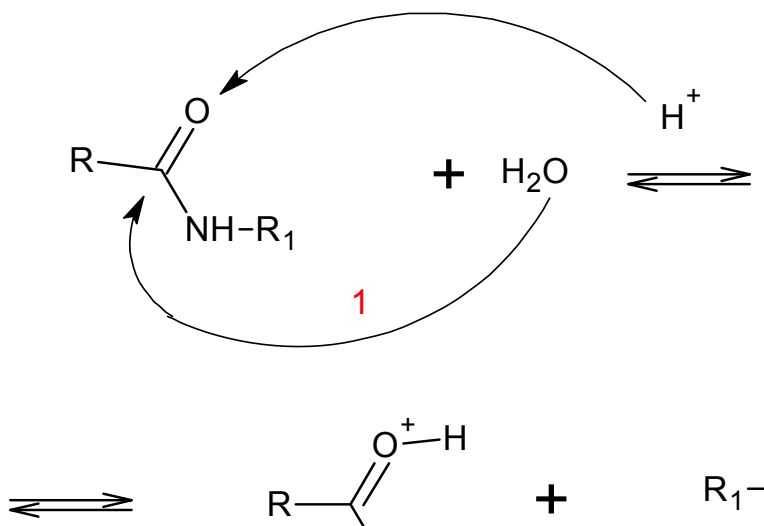<smiles>[R]C(O)=[OH+]</smiles>

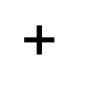

$\mathrm{R}_{1}-\mathrm{NH}_{2}$

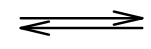<smiles>C=CC</smiles><smiles>[R]C(=O)O</smiles><smiles>[R7][NH+]([CH])[C@H](O)C([R])(O)O</smiles>

2<smiles>[3H+]</smiles>

Fig. 1: Mecanismo de la Hidrólisis Ácida

Las estructuras de todos los reactivos y productos fueron optimizadas y en la Tabla 1 se reportan los valores de longitudes de enlace, ángulos de enlace, ángulos diedros obtenidos como resultado de la aplicación del método DFT y las energías (E) expresadas en $\mathrm{kcal} / \mathrm{mol}$ calculadas mediante el método AM1, de los reactivos y productos a saber: Acetamida, N-metilacetamida, Benzamida, N-metilbenzamida, metilamina, ácido acético y ácido benzoico, que intervienen en cada una de las reacciones motivo de estudio. Además se obtuvieron, mediante la aplicación del método AM1, las longitudes de enlaces, ángulos de enlaces y energías de cada uno de los intermediarios de reacción de la hidrólisis ácida de cada una de las amidas alifáticas y aromáticas en estudio y dichos valores se muestran en la Tabla 2.

Se calcularon las energías de activación (Ea) de cada una de las reacciones. Las mismas resultan de la diferencia de energía entre la etapa 1 (especie protonada) y la etapa 2 (intermediario de reacción), etapas indicadas en el esquema general del mecanismo de reacción. Para la hidrólisis ácida de Acetamida y Nmetilacetamida se obtuvieron energías de activación de $18.83 \mathrm{kcal} / \mathrm{mol}$ y $20.74 \mathrm{kcal} / \mathrm{mol}$ respectivamente, valores que se aproximan a los reportados de $19.8 \mathrm{kcal} / \mathrm{mol}$ (Bolton, 1965) y de $21.5 \mathrm{kcal} / \mathrm{mol}$ (Bolton, 1966), respectivamente. Para la hidrólisis ácida de Benzamida y $\mathrm{N}$-metilbenzamida se obtuvieron valores de energías de activación de $21,47 \mathrm{kcal} / \mathrm{mol}$ y $22.74 \mathrm{kcal} / \mathrm{mol}$, respectivamente, que se aproximan a los valores reportados de $21.00 \mathrm{kcal} / \mathrm{mol}$ y $21.62 \mathrm{kcal} / \mathrm{mol}$ (Cox y Yates, 198), respectivamente. La mayor reactividad observada en las amidas alifáticas se puede asociar a que la deficiencia electrónica que posee el carbono del carbonilo, luego de la protonación del oxígeno del carbonilo, está compensada por el efecto inductivo $\mathrm{sp}^{3} \rightarrow \mathrm{sp}^{2}$ ejercido por el grupo metilo, mientras que en el caso de las amidas aromáticas, la densidad de carga positiva puede deslocalizarse en el anillo aromático. Esta deslocalización de la carga compensa más la deficiencia electrónica que el efecto inductivo debido a la diferencia de hibridación, por lo tanto en el caso de las amidas alifáticas, el carbono del carbonilo está más susceptible a ser atacado por el agente nucleofílico, siendo por lo tanto mayor la reactividad de estas amidas frente a la hidrólisis catalizada por ácidos, en comparación con las amidas aromáticas. 
Tabla 1: Parámetros Geométricos y Energías de Reactivos y Productos

\begin{tabular}{|c|c|c|c|c|c|}
\hline & Estructuras & $\begin{array}{l}\text { Longitudes de } \\
\text { Enlace }(\AA)\end{array}$ & Ángulos de Enlace $\left(^{\circ}\right)$ & Ángulos Diedros $\left({ }^{\circ}\right)$ & $E$ \\
\hline Acetamida & & $\begin{array}{l}\mathrm{C}_{1}-\mathrm{C}_{6}=1.51 \\
\mathrm{C}_{1}-\mathrm{O}=1.25 \\
\mathrm{C}_{1}-\mathrm{N}=1.37 \\
\mathrm{~N}-\mathrm{H}=0.98\end{array}$ & $\begin{array}{l}\mathrm{C}_{6}-\mathrm{C}_{1}-\mathrm{N}=118.17 \\
\mathrm{O}-\mathrm{C}_{1}-\mathrm{N}=119.83 \\
\mathrm{C}_{1}-\mathrm{N}-\mathrm{H}_{5}=121.19 \\
\mathrm{C}_{1}-\mathrm{N}-\mathrm{H}_{4}=119.77\end{array}$ & $\begin{array}{l}\mathrm{H}_{5}-\mathrm{N}_{3}-\mathrm{C}_{1}-\mathrm{O}_{2}=179.85 \\
\mathrm{H}_{7}-\mathrm{C}_{6}-\mathrm{C}_{1}-\mathrm{O}_{2}=119.47\end{array}$ & -50.76 \\
\hline $\begin{array}{c}\text { Trans- } \\
\text { N-metil } \\
\text { acetamida }\end{array}$ & & $\begin{array}{l}\mathrm{C}_{1}-\mathrm{C}_{5}=1.51 \\
\mathrm{C}_{1}-\mathrm{O}=1.25 \\
\mathrm{C}_{1}-\mathrm{N}=1.38 \\
\mathrm{~N}-\mathrm{H}=0.99 \\
\mathrm{~N}-\mathrm{C}_{9}=1.42\end{array}$ & $\begin{array}{c}\mathrm{C}_{5}-\mathrm{C}_{1}-\mathrm{N}=117.26 \\
\mathrm{O}-\mathrm{C}_{1}-\mathrm{N}=121.23 \\
\mathrm{C}_{1}-\mathrm{N}-\mathrm{C}_{9}=123.14 \\
\mathrm{H}-\mathrm{N}-\mathrm{C}_{9}=117.76\end{array}$ & $\begin{array}{l}\mathrm{H}_{4}-\mathrm{N}_{3}-\mathrm{C}_{1}-\mathrm{O}_{2}=179.99 \\
\mathrm{H}_{6}-\mathrm{C}_{5}-\mathrm{C}_{1}-\mathrm{O}_{2}=119.54 \\
\mathrm{H}_{10}-\mathrm{C}_{9}-\mathrm{N}_{3}-\mathrm{C}_{1}=120.18\end{array}$ & -47.36 \\
\hline $\begin{array}{l}\text { Cis- N-metil } \\
\text { acetamida }\end{array}$ & & $\begin{array}{l}\mathrm{C}_{1}-\mathrm{C}_{4}=1.51 \\
\mathrm{C}_{1}-\mathrm{O}=1.25 \\
\mathrm{C}_{1}-\mathrm{N}=1.38 \\
\mathrm{~N}-\mathrm{H}=0.99 \\
\mathrm{~N}-\mathrm{C}_{8}=1.43\end{array}$ & $\begin{array}{c}\mathrm{C}_{4}-\mathrm{C}_{1}-\mathrm{N}=118.56 \\
\mathrm{O}-\mathrm{C}_{1}-\mathrm{N}=120.48 \\
\mathrm{C}_{1}-\mathrm{N}-\mathrm{C}_{8}=120.93 \\
\mathrm{H}-\mathrm{N}-\mathrm{C}_{8}=121.93\end{array}$ & $\begin{array}{l}\mathrm{C}_{4}-\mathrm{C}_{1}-\mathrm{O}_{2}-\mathrm{N}_{3}=178.81 \\
\mathrm{H}_{5}-\mathrm{C}_{4}-\mathrm{C}_{1}-\mathrm{O}_{2}=126.65 \\
\mathrm{H}_{11}-\mathrm{C}_{8}-\mathrm{N}_{3}-\mathrm{C}_{1}=70.84\end{array}$ & -46.46 \\
\hline Benzamida & & $\begin{array}{l}\mathrm{C}_{1}-\mathrm{C}_{6}=1.49 \\
\mathrm{C}_{1}-\mathrm{O}=1.25 \\
\mathrm{C}_{1}-\mathrm{N}=1.37 \\
\mathrm{~N}-\mathrm{H}=0.98\end{array}$ & $\begin{array}{l}\mathrm{C}_{6}-\mathrm{C}_{1}-\mathrm{N}=118.33 \\
\mathrm{O}-\mathrm{C}_{1}-\mathrm{N}=119.72 \\
\mathrm{C}_{1}-\mathrm{N}-\mathrm{H}_{5}=121.21 \\
\mathrm{C}_{1}-\mathrm{N}-\mathrm{H}_{4}=119.38\end{array}$ & $\begin{array}{l}\mathrm{C}_{6}-\mathrm{C}_{1}-\mathrm{O}_{2}-\mathrm{N}_{3}=179.45 \\
\mathrm{C}_{7}-\mathrm{C}_{6}-\mathrm{C}_{1}-\mathrm{O}_{2}=144.87 \\
\mathrm{H}_{5}-\mathrm{N}_{3}-\mathrm{C}_{1}-\mathrm{O}_{2}=175.31 \\
\mathrm{H}_{13}-\mathrm{C}_{8}-\mathrm{C}_{7}-\mathrm{C}_{6}=179.76\end{array}$ & -16.21 \\
\hline $\begin{array}{c}\text { Trans- } \\
\mathrm{N} \text {-metil } \\
\text { benzamida }\end{array}$ & & $\begin{array}{c}\mathrm{C}_{1}-\mathrm{C}_{4}=1.49 \\
\mathrm{C}_{1}-\mathrm{O}=1.25 \\
\mathrm{C}_{1}-\mathrm{N}=1.38 \\
\mathrm{~N}-\mathrm{H}=0.99 \\
\mathrm{~N}-\mathrm{C}_{15}=1.43\end{array}$ & $\begin{array}{c}\mathrm{C}_{4}-\mathrm{C}_{1}-\mathrm{N}=117.31 \\
\mathrm{O}-\mathrm{C}_{1}-\mathrm{N}=121.17 \\
\mathrm{C}_{1}-\mathrm{N}-\mathrm{C}_{15}=122.85 \\
\mathrm{H}-\mathrm{N}-\mathrm{C}_{15}=117.79\end{array}$ & $\begin{array}{l}\mathrm{C}_{4}-\mathrm{C}_{1}-\mathrm{O}_{2}-\mathrm{N}_{3}=179.55 \\
\mathrm{C}_{5}-\mathrm{C}_{4}-\mathrm{C}_{1}-\mathrm{O}_{2}=141.69 \\
\mathrm{H}_{11}-\mathrm{C}_{6}-\mathrm{C}_{5}-\mathrm{C}_{4}=179.75 \\
\mathrm{H}_{13}-\mathrm{C}_{8}-\mathrm{C}_{7}-\mathrm{C}_{6}=179.47\end{array}$ & -12.76 \\
\hline $\begin{array}{l}\text { Cis- N-metil } \\
\text { benzamida }\end{array}$ & & $\begin{array}{c}\mathrm{C}_{1}-\mathrm{C}_{5}=1.49 \\
\mathrm{C}_{1}-\mathrm{O}=1.25 \\
\mathrm{C}_{1}-\mathrm{N}=1.38 \\
\mathrm{~N}-\mathrm{H}=0.99 \\
\mathrm{~N}-\mathrm{C}_{16}=1.42\end{array}$ & $\begin{array}{c}\mathrm{C}_{5}-\mathrm{C}_{1}-\mathrm{N}=120.15 \\
\mathrm{O}-\mathrm{C}_{1}-\mathrm{N}=118.42 \\
\mathrm{C}_{1}-\mathrm{N}-\mathrm{C}_{16}=125.62 \\
\mathrm{H}-\mathrm{N}-\mathrm{C}_{16}=117.54\end{array}$ & $\begin{array}{l}\mathrm{C}_{5}-\mathrm{C}_{1}-\mathrm{O}_{2}-\mathrm{N}_{3}=179.43 \\
\mathrm{C}_{6}-\mathrm{C}_{5}-\mathrm{C}_{1}-\mathrm{O}_{2}=128.89 \\
\mathrm{H}_{12}-\mathrm{C}_{7}-\mathrm{C}_{6}-\mathrm{C}_{5}=179.63 \\
\mathrm{H}_{14}-\mathrm{C}_{9}-\mathrm{C}_{8}-\mathrm{C}_{7}=179.54\end{array}$ & -11.36 \\
\hline Metilamina & & $\begin{array}{l}\mathrm{C}-\mathrm{H}=1.12 \\
\mathrm{C}-\mathrm{N}=1.43 \\
\mathrm{~N}-\mathrm{H}=1.00\end{array}$ & $\begin{array}{l}\mathrm{H}-\mathrm{N}-\mathrm{H}=109.05 \\
\mathrm{H}-\mathrm{C}-\mathrm{N}=109.01 \\
\mathrm{C}-\mathrm{N}-\mathrm{H}=111.31\end{array}$ & $\begin{array}{l}\mathrm{C}_{4}-\mathrm{N}_{1}-\mathrm{H}_{2}-\mathrm{H}_{3}=123.19 \\
\mathrm{H}_{5}-\mathrm{C}_{4}-\mathrm{N}_{1}-\mathrm{H}_{2}=178.12\end{array}$ & -7.42 \\
\hline $\begin{array}{l}\text { Ácido } \\
\text { Acético }\end{array}$ & 3 & $\begin{array}{l}\mathrm{C}-\mathrm{O}_{2}=1.23 \\
\mathrm{C}-\mathrm{C}=1.49 \\
\mathrm{C}-\mathrm{O}_{3}=1.36 \\
\mathrm{H}-\mathrm{O}_{3}=0.97 \\
\mathrm{C}-\mathrm{H}=1.12\end{array}$ & $\begin{array}{l}\mathrm{C}-\mathrm{O}-\mathrm{H}=109.76 \\
\mathrm{O}-\mathrm{C}-\mathrm{O}=116.53 \\
\mathrm{O}_{2}-\mathrm{C}-\mathrm{C}=129.27 \\
\mathrm{O}_{3}-\mathrm{C}-\mathrm{C}=114.20 \\
\mathrm{C}-\mathrm{C}-\mathrm{H}_{7}=108.94\end{array}$ & $\begin{array}{l}\mathrm{C}_{5}-\mathrm{C}_{1}-\mathrm{O}_{2}-\mathrm{O}_{3}=179.99 \\
\mathrm{H}_{8}-\mathrm{C}_{5}-\mathrm{C}_{1}-\mathrm{O}_{2}=179.98 \\
\mathrm{H}_{7}-\mathrm{C}_{5}-\mathrm{C}_{1}-\mathrm{O}_{2}=59.35\end{array}$ & -103.8 \\
\hline $\begin{array}{c}\text { Ácido } \\
\text { Benzoico }\end{array}$ & & $\begin{array}{l}\mathrm{C}_{12}-\mathrm{O}_{13}=1.24 \\
\mathrm{C}_{12}-\mathrm{O}_{14}=1.37 \\
\mathrm{C}_{12}-\mathrm{C}_{3}=1.47 \\
\mathrm{O}_{14}-\mathrm{H}_{15}=0.97 \\
\mathrm{C}_{2}-\mathrm{H}_{8}=1.10\end{array}$ & $\begin{array}{c}\mathrm{O}_{13}-\mathrm{C}_{12}-\mathrm{O}_{14}=116.02 \\
\mathrm{C}_{12}-\mathrm{O}_{14}-\mathrm{H}_{15}=109.07 \\
\mathrm{O}_{13}-\mathrm{C}_{12}-\mathrm{C}_{3}=128.62 \\
\mathrm{H}_{8}-\mathrm{C}_{2}-\mathrm{C}_{3}=119.40\end{array}$ & $\begin{array}{l}\mathrm{O}_{14}-\mathrm{C}_{12}-\mathrm{C}_{3}-\mathrm{C}_{2}=179.9 \\
\mathrm{H}_{8}-\mathrm{C}_{2}-\mathrm{C}_{1}-\mathrm{C}_{6}=179.99 \\
\mathrm{H}_{9}-\mathrm{C}_{4}-\mathrm{C}_{3}-\mathrm{C}_{2}=179.98\end{array}$ & -68.10 \\
\hline
\end{tabular}


Tabla 2: Parámetros Geométricos y Energías de Intermediarios Alifáticos y Aromáticos

\begin{tabular}{|c|c|c|c|c|}
\hline $\begin{array}{l}\text { Intermediarios } \\
\text { Hidrólisis }\end{array}$ & Estructuras & $\begin{array}{l}\text { Longitudes de } \\
\text { Enlace }(\AA)\end{array}$ & Ángulos de Enlace $\left(^{\circ}\right)$ & $\begin{array}{l}\text { Energías } \\
\text { kcal mol }^{-1}\end{array}$ \\
\hline Acetamida & & $\begin{array}{c}\mathrm{C}_{1}-\mathrm{O}_{4}=1.40 \\
\mathrm{C}_{1}-\mathrm{N}_{10}=1.42 \\
\mathrm{C}_{1}-\mathrm{C}_{6}=1.52 \\
\mathrm{~N}_{10}-\mathrm{H}_{11}=1.00 \\
\mathrm{O}_{2}-\mathrm{H}_{3}=0.96 \\
\mathrm{C}_{6}-\mathrm{H}_{9}=1.11 \\
\mathrm{O}_{4}-\mathrm{H}_{5}=0.97 \\
\mathrm{C}_{1}-\mathrm{O}_{2}=1.42\end{array}$ & $\begin{array}{c}\mathrm{C}_{1}-\mathrm{O}_{4}-\mathrm{H}_{5}=112.31 \\
\mathrm{C}_{1}-\mathrm{N}_{10}-\mathrm{H}_{11}=115.86 \\
\mathrm{H}_{8}-\mathrm{C}_{6}-\mathrm{H}_{9}=109.44 \\
\mathrm{C}_{1}-\mathrm{O}_{2}-\mathrm{H}_{13}=120.57 \\
\mathrm{C}_{6}-\mathrm{C}_{1}-\mathrm{O}_{4}=107.33 \\
\mathrm{C}_{6}-\mathrm{C}_{1}-\mathrm{N}_{10}=126.26 \\
\mathrm{O}_{4}-\mathrm{C}_{1}-\mathrm{O}_{2}=103.99 \\
\mathrm{C}_{6}-\mathrm{C}_{1}-\mathrm{O}_{2}=112.77\end{array}$ & 104.07 \\
\hline $\mathrm{N}$-metilacetamida & & $\begin{array}{c}\mathrm{O}_{4}-\mathrm{H}_{16}=0.91 \\
\mathrm{C}_{1}-\mathrm{O}_{4}=1.40 \\
\mathrm{C}_{1}-\mathrm{O}_{2}=1.42 \\
\mathrm{C}_{1}-\mathrm{C}_{5}=1.53 \\
\mathrm{C}_{1}-\mathrm{N}_{9}=1.46 \\
\mathrm{O}_{2}-\mathrm{H}_{3}=0.96 \\
\mathrm{C}_{12}-\mathrm{N}_{9}=1.39 \\
\mathrm{C}_{12}-\mathrm{H}_{13}=1.07 \\
\mathrm{C}_{5}-\mathrm{H}_{6}=1.11 \\
\mathrm{~N}_{9}-\mathrm{H}_{10}=1.02\end{array}$ & $\begin{array}{c}\mathrm{H}_{16}-\mathrm{O}_{4}-\mathrm{C}_{1}=119.83 \\
\mathrm{C}_{5}-\mathrm{C}_{1}-\mathrm{O}_{2}=112.77 \\
\mathrm{~N}_{9}-\mathrm{C}_{1}-\mathrm{O}_{2}=104.09 \\
\mathrm{H}_{7}-\mathrm{C}_{5}-\mathrm{C}_{1}=110.14 \\
\mathrm{C}_{1}-\mathrm{O}_{2}-\mathrm{H}_{11}=120.33 \\
\mathrm{C}_{5}-\mathrm{C}_{1}-\mathrm{N}_{9}=126.26 \\
\mathrm{H}_{15}-\mathrm{C}_{12}-\mathrm{N}_{9}=109.49 \\
\mathrm{C}_{12}-\mathrm{N}_{9}-\mathrm{H}_{10}=118.14 \\
\mathrm{C}_{1}-\mathrm{N}_{2}-\mathrm{C}_{12}=120.84 \\
\mathrm{C}_{5}-\mathrm{C}_{1}-\mathrm{O}_{4}=107.33\end{array}$ & 118.69 \\
\hline Benzamida & & $\begin{array}{l}\mathrm{C}_{1}-\mathrm{O}_{2}=1.42 \\
\mathrm{C}_{1}-\mathrm{N}_{3}=1.46 \\
\mathrm{C}_{1}-\mathrm{C}_{6}=1.52 \\
\mathrm{~N}_{3}-\mathrm{H}_{4}=1.01 \\
\mathrm{O}_{2}-\mathrm{H}_{20}=0.97 \\
\mathrm{C}_{6}-\mathrm{C}_{7}=1.40 \\
\mathrm{O}_{17}-\mathrm{H}_{18}=0.94 \\
\mathrm{C}_{1}-\mathrm{O}_{17}=1.43\end{array}$ & $\begin{array}{c}\mathrm{C}_{1}-\mathrm{O}_{2}-\mathrm{H}_{20}=115.86 \\
\mathrm{C}_{1}-\mathrm{N}_{3}-\mathrm{H}_{4}=115.26 \\
\mathrm{H}_{18}-\mathrm{O}_{17}-\mathrm{C}_{1}=115.82 \\
\mathrm{O}_{17}-\mathrm{C}_{1}-\mathrm{O}_{2}=100.85 \\
\mathrm{O}_{2}-\mathrm{C}_{1}-\mathrm{N}_{3}=110.87 \\
\mathrm{O}_{17}-\mathrm{C}_{1}-\mathrm{N}_{3}=110.50 \\
\mathrm{~N}_{3}-\mathrm{C}_{1}-\mathrm{C}_{6}=111.93 \\
\mathrm{C}_{1}-\mathrm{C}_{6}-\mathrm{C}_{7}=120.62\end{array}$ & 124.60 \\
\hline $\begin{array}{c}\mathrm{N} \text {-metil } \\
\text { benzamida }\end{array}$ & . & $\begin{array}{c}\mathrm{O}_{2}-\mathrm{H}_{23}=0.96 \\
\mathrm{C}_{1}-\mathrm{N}_{3}=1.46 \\
\mathrm{C}_{1}-\mathrm{O}_{2}=1.43 \\
\mathrm{~N}_{3}-\mathrm{H}_{4}=1.00 \\
\mathrm{~N}_{3}-\mathrm{C}_{16}=1.44 \\
\mathrm{C}_{16}-\mathrm{H}_{17}=1.12 \\
\mathrm{C}_{1}-\mathrm{C}_{5}=1.52 \\
\mathrm{C}_{1}-\mathrm{O}_{21}=1.42 \\
\mathrm{C}_{6}-\mathrm{H}_{11}=1.10 \\
\mathrm{O}_{21}-\mathrm{H}_{22}=0.97\end{array}$ & $\begin{array}{c}\mathrm{C}_{1}-\mathrm{O}_{2}-\mathrm{H}_{23}=119.09 \\
\mathrm{C}_{1}-\mathrm{N}_{3}-\mathrm{C}_{16}=115.45 \\
\mathrm{C}_{6}-\mathrm{C}_{5}-\mathrm{C}_{1}=120.23 \\
\mathrm{H}_{18}-\mathrm{C}_{16}-\mathrm{N}_{3}=108.43 \\
\mathrm{O}_{2}-\mathrm{C}_{1}-\mathrm{N}_{3}=109.58 \\
\mathrm{H}_{22}-\mathrm{O}_{21}-\mathrm{C}_{1}=108.33 \\
\mathrm{C}_{5}-\mathrm{C}_{1}-\mathrm{N}_{3}=111.11 \\
\mathrm{O}_{21}-\mathrm{C}_{1}-\mathrm{N}_{3}=114.14 \\
\mathrm{O}_{21}-\mathrm{C}_{1}-\mathrm{O}_{2}=102.52 \\
\mathrm{O}_{11}-\mathrm{C}_{6}-\mathrm{C}_{5}=119.52\end{array}$ & 124.71 \\
\hline
\end{tabular}

La mayor reactividad de Acetamida frente a la hidrólisis ácida en comparación con la N-metilacetamida se asocia al reemplazo de un hidrógeno del nitrógeno por un grupo metilo, grupo más voluminoso que produce un mayor impedimento estéreo, dificultando el ataque del nucleófilo $\left(\mathrm{H}_{2} \mathrm{O}\right)$ al carbono del carbonilo, provocando una menor estabilidad del intermediario y un aumento en la correspondiente energía de activación. Por el mismo motivo la benzamida reportó mayor reactividad que la metilbenzamida frente a la hidrólisis catalizada por ácidos. La mayor reactividad de Acetamida frente a la hidrólisis ácida en comparación con Benzamida se asocia a la presencia del anillo bencénico, que al ser un grupo más voluminoso, ocasiona un mayor impedimento estérico y los cálculos de energía a nivel teórico indican que el intermediario alifático es más estable que el intermediario aromático.

Como complemento del estudio teórico se realizaron experimentalmente las hidrólisis catalizadas por ácido de las siguientes amidas: Acetamida, N-metilacetamida y Benzamida. Las mismas se llevaron a cabo en un microreactor sumergido en un baño termostatizado a $80^{\circ} \mathrm{C}$, equipado con un condensador a reflujo y agitación magnética durante $2 \mathrm{~h}$. Se trabajó con ácido sulfúrico, como catalizador, con una concentración $0.09 \mathrm{M}$ y una concentración de $0.1 \mathrm{M}$ para cada una de las amidas reactivos. Los productos se analizaron y cuantificaron en un cromatógrafo gaseoso HP 5890 acoplado a un detector selectivo de masa HP 5972. La 
conversión del sustrato se midió como función del tiempo de reacción. Los valores de conversión, frente a la hidrólisis ácida, de Acetamida, N-metilacetamida y Benzamida fueron 92.11, 41.56 y 24,39 moles \% respectivamente.

\section{CONCLUSIONES}

De acuerdo a los resultados y discusión presentados en este trabajo, se pueden obtener las siguientes conclusiones:

i) Los intermediarios tetraédricos alifáticos resultan ser más estables que los aromáticos, por lo tanto las amidas alifáticas presentan una mayor reactividad frente a la hidrólisis ácida, comparada con las amidas aromáticas, presentando estas últimas una menor velocidad de reacción. La facilidad con que las amidas alifáticas se hidrolizan coincide con la aplicación de esta reacción en la degradación de poliamidas.

ii) La existencia de una marcada concordancia entre los resultados teóricos, datos experimentales y de literatura, referidos a la reactividad de las amidas alifáticas y aromáticas frente a la hidrólisis ácida, valida el empleo de los métodos teóricos DFT y AM1 como herramientas para el estudio de la estructura de los reactivos y productos que participan del sistema químico planteado y para estimar la cinética del mismo.

\section{REFERENCIAS}

Becke, A.D., Density-functional thermochemistry V. Systematic optimization of exchange-correlation functionals, J. Chem. Phys.: 107, 8554-8560 (1997).

Bolton, P.D., Hydrolysis of Amides.II.Substituent effects in dilute acid and alkali, Aust. J. Chem.:19,10131021 (1966).

Bolton, P.D. y I.R. Wilson, Hydrolysis of Amides.I. The hydrolysis of acetamide and chloroacetamide in dilute acid, Aust. J. Chem.:18, 795-800 (1965).

Cox, R.A. y K. Yates, The Hydrolysis of benzamides, methylbenzimidatium ions, and lactams in aqueous sulfuric acid. The excess acidity method in the determination of reaction mechanisms, Can.J.Chem.:59,28532863 (1981).

Cheshmedzhieva, D., S. Hieva, B.Hadjieva, T. Trayanova y B. Galabov, Reactivity of acetanilides in the alkaline hydrolysis reaction:theory vs. experiment, Molecular Physics:107,1187-1192 (2009).

Dewar, M.J.S., E.G.Zoebisch, E.F.Healy y J.J.P.Stewart, AM1: A New General Purpose Quantum Mechanical Molecular Model, J. Am. Chem.Soc.:107, 3902-3909 (1985).

Frisch, M.J., G. W. Trucks, y otros 80 autores; Gaussian 03, Revision E.01; Gaussian, Inc., Wallingford CT, Pittsburgh, USA (2004).

Hori, K., Y. Ikenaga, K. Arata, T. Takahashi, K. Kasai, Y. Noguchi, M. Sumimoto y H. Yamamoto, Theoretical study on the reaction mechanism for the hydrolysis of esters and amides under acidic conditions, Tetrahedron: 63, 1264-1269 (2007).

Jacques, B.,M. Werth, I. Merdas, F. Thominette y J. Verdu, Hydrolytic ageing of polyamide 11.1. Hydrolysis kinetics in water, Polymer: 43, 6439-6447 (2002).

Morero, B. y E. A. Campanella, Simulation of the Process of Chemical Absorption using Amine Solutions for Biogas Purification, Inf. Tecnol.:24(1), 25-32 (2013).

Parr, R.G. y W. Yang, Density-functional theory of atoms and molecules, Oxford University Press.-Nueva York (1989).

Wang, L., Y. Wang y D. Cao, Synthesis and Characterization of Novel Biodegradable Polyamides Containing $\alpha$-amino Acid,Journal of Macromolecular Science, Part A: Pure and Applied Chemistry: 46, 312-320 (2009).

Xiong, Y. y C.G. Zhan, Theoretical Studies of the Transition-State Structures and Free Energy Barriers for Base-Catalyzed Hydrolysis of Amides, J.Phys.Chem.A.: 110, 12644-12652 (2006). 